Indexed by

\title{
Scopus
}

\section{PREDICTION OF ENERGY CONSUMPTION IN THE LEADWELL V-40 IT CNC MACHINING CENTER THROUGH ARTIFICIAL NEURAL NETWORKS}

Crossref

KOBSON

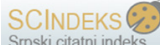

81Google

\section{M.A. Rodriguez-Cabal}

Instituto Tecnológico Metropolitano, Faculty of Engineering, Medellín, Colombia

\author{
Juan Gonzalo Ardila Marín \\ Universidad Surcolombiana, \\ Faculty of Engineering, \\ Neiva, \\ Colombia
}

\author{
Sebastián Rudas \\ Institución Universitaria \\ Pascual Bravo, Faculty of \\ Engineering, Medellin, \\ Colombia
}

Key words: neural networks, CNC, energy management, manufacture industry doi:10.5937/jaes0-30826

Cite article:

A. Rodriguez-Cabal M., Gonzalo Ardila Marín J., Rudas S. (2022) PREDICTION OF ENERGY CONSUMPTION IN THE LEADWELL V-40 IT CNC MACHINING CENTER THROUGH ARTIFICIAL NEURAL NETWORKS, Journal of Applied Engineering Science, 20(1), 145 - 149, DOI:10.5937/ jaes0-30826

Online aceess of full paper is available at: www.engineeringscience.rs/browse-issues 


\title{
PREDICTION OF ENERGY CONSUMPTION IN THE LEADWELL V-40 IT CNC MACHINING CENTER THROUGH ARTIFICIAL NEURAL NETWORKS
}

\author{
M.A. Rodriguez-Cabal' ${ }^{*}$, Juan Gonzalo Ardila Marín', Sebastián Rudas ${ }^{3}$ \\ 1 Instituto Tecnológico Metropolitano, Faculty of Engineering, Medellín, Colombia \\ ${ }^{2}$ Universidad Surcolombiana, Faculty of Engineering, Neiva, Colombia \\ ${ }^{3}$ Institución Universitaria Pascual Bravo, Faculty of Engineering, Medellin, Colombia
}

\begin{abstract}
Energy consumption in machining processes has become a problem for today's manufacturing industry. The use of neural networks and optimization algorithms for modeling and prediction of consumption as a function of the cut-off parameters in processes of this type has aroused the interest of research groups. The present work used artificial neural networks (ANN) to predict the energy consumption of a Leadwell V-40iT ${ }^{\circledR}$ five-axis CNC machining center, based on experimental data obtained through a factorial experimental design 53. ANN was programed in Matlab ${ }^{\circledR}$. From the study was concluded that the depth per pass (Ap) is the variable that has the most influence on the prediction model of energy consumption with a $77 \%$ of relative importance, while the feed rate is the least relevant with $9 \%$ of importance.
\end{abstract}

Key words: neural networks, CNC, energy management, manufacture industry

\section{INTRODUCTION}

Energy consumption in the manufacturing industry, being the main axis of many companies, has been studied with the aim of improving energy efficiency and profitability. With the arrival of new technologies such as machine tools with computerized numerical control (CNC), automation of processes has been achieved, reducing manufacturing times and costs. Based on this, various methods have been explored to achieve a reduction in energy and material consumption from the optimization of machining processes. Among the main manipulated cutting variables such as feed speed, spindle speed, and radial and axial depth of cut, and the definition of the strategies that determine the tool paths to carry out the machining. In the work developed by $\mathrm{Hu}$, et al [1], energy consumption is minimized by optimizing machining sequences, by reducing the number of machine positionings and achieving a $14 \%$ reduction in consumption and $21 \%$ in the time of machining. On the other hand, in other studies, the multi-objective optimization of the parameters and trajectories is developed, managing to reduce the energy consumption of the machine by $16 \%$ and a reduction in machining time [2]. Another study is the realized by $\mathrm{Ma}$, et al, where a theoretical mathematical model was used to minimize energy consumption by controlling the speed of the spindle, the authors found the appropriate speeds that did not affect the piece finishing and that in turn presented energy savings. In the same way, they carried out the validation of the model with real experimentation and had an $80 \%$ approximation between the theoretical value of savings and the experimental on [3].

Specialized literature presents multiple theoretical models trying to obtain a good approximation to the real pro- cess, however, their error margins are still very high [4], [5] . Such is the case presented by Mori, et al, who made the evaluation of the spindle acceleration. Yan and $\mathrm{Li}$, evaluate the impact of the rotational speed, feed rate, axial depth and radial depth in the superficial finishing in a turning process. additionally, in the specialized literature, there are works in which they perform the optimization of the machining processes or made the evaluation of the surface finishing taking into account as main cutting parameters the spindle speed, feed rate and axial depth [6]-[10].

On the other hand, the implementation of predictive models through neural networks which are based on real data helps to reduce errors and improve the prediction of energy consumption, surface finish, or cutting forces, thus having more efficient processes [11], [12]. Such is the case presented by Vishnu, et al, where they performed an optimization of the cutting parameters and prediction of the surface roughness for machining a P20 steel by means of neural networks. Where they implement the Taguchi methodology to reduce the number of experiments required, for neural networks they use a multilayer model of nonlinear mapping. The authors report a correlation of 1 between experimental and theoretical data which means that the AAN purposed can be implemented to know the surface finish of machining of a P20 steel [8]. Moreover, Zerti, et al, carry out a study to predict the machining performance of the machining process of a martensitic steel by means of neural networks using as input variables the main cutting variables and considering the roughness and cutting forces exerted in all directions, the authors obtain an R2 of $95 \%$ which is an acceptable value, finding that the feed rate is the vari- 
able that most influences the surface finish [9].

In the specialized literature, the use of neural networks to predict the surface finish which is related to cutting parameters that minimize roughness and cutting forces, has been successfully implemented, which are important to improve the quality of the processes. However, there are few studies focused on the energy consumption of machine tools, which is a variable of importance in the manufacturing industry, for this reason is important to determine the cutting parameters that allow more efficient machining processes. It is for the above that in this paper, a model based on ANN was designed, programmed, and validated to predict the energy consumption of a Leadwell V-40iT® five-axis CNC machining center. For the construction of the database for the training and validation process, an experiment was carried out in the Leadwell V-40iT® machining center. Using an experimental factorial design that included spindle speed (Vs), feed rate (Vf) and cutting depth (Ap).

\section{EXPERIMENTAL DATA}

To obtain the database, a factorial experimental design 53 was implemented, which allows having a broader measurement spectrum, which translates into the acquisition of more detailed experimental data, considering intermediate points of the main cutting variables. The data is based on the operating recommendation of the tool manufacturer, which was a $1 / 2$ " Master Cut $\circledast$ cylindrical bur. Table 1 shows the parameters implemented for the 125 experiments, which were performed on an AISI 1045 steel plate with a radial cutting depth of $75 \%$. As is mentioned above, in this study was taken as main cutting parameters the $\mathrm{Vs}, \mathrm{Vf}$ and $\mathrm{Ap}$, because they are the variables that are mainly controlled in the works published in the specialized literature and have a direct influence on energy consumption and surface finishing. Is important to highlight that the radial depth and refrigerant depends on the tool manufacturer recommendation and the type of workpiece material. The objective of the experimental design is the measurement of the energy consumption associated with the different configurations mentioned in a measurement range, which will be the output of the neural network, in Figure 1 a schematic representation of the taking of variables that have an effect in the machining process and related output.

Table 1: Parameters of factorial design

\begin{tabular}{|c|c|}
\hline Cutting parameter & Range \\
\hline Spindle speed $\left(V_{s}\right)$ & $950-1900 \mathrm{rpm}$ \\
\hline Feed rate $\left(\mathrm{V}_{\mathrm{f}}\right)$ & $134-268 \mathrm{~mm} / \mathrm{min}$ \\
\hline Cut depth $\left(\mathrm{A}_{\mathrm{o}}\right)$ & $1-3 \mathrm{~mm}$ \\
\hline
\end{tabular}

\section{ARTIFICIAL NEURAL NETWORK}

Artificial neural networks (ANN) are mathematical approximation models where inputs are related to a specific output, all through the analysis of a set of experimental

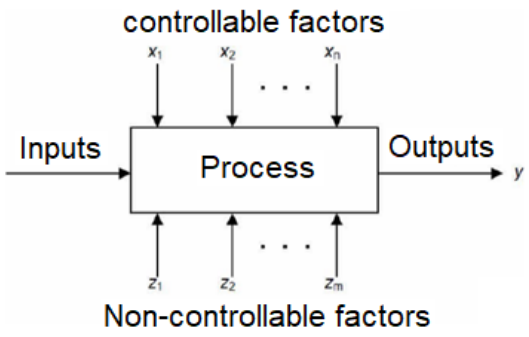

Figure 1: Schematic representation of experimental design

data. From this analysis, correlations are found to define the architecture of the network. The prediction of the output, or target, is performed if the input is within the limits of the database with which the training was carried out [13]. In the present work, a feed-forward network type multilayer neural network model was developed, which has one or more hidden layers of neurons with sigmoid function followed by a layer of linear neurons, this architecture allows establishing linear and not linear transfer functions, providing adjustments in a better way to the studied phenomena [14]. In Figure 2 the implemented architecture is shown, there you can see the number of inputs, the hidden layer with the transfer function where the weights are assigned to each of the variables and their adjustment (bias), and finally the layer linear output with an additional adjustment component corresponding to the linear function [15].

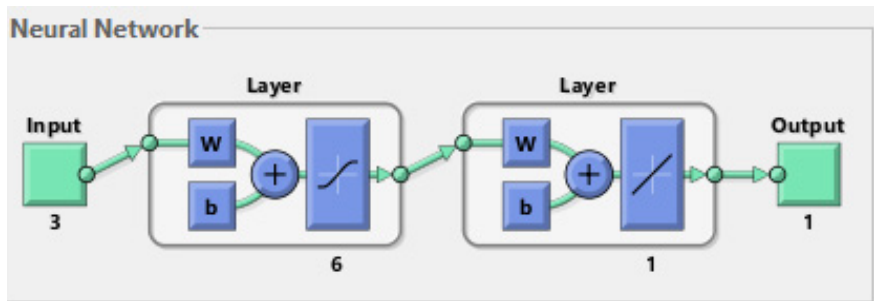

Figure 2: ANN architecture [9]

For this work, the hyperbolic tangent sigmoid function was implemented as a transfer function, accompanied by the PURELIN output layer, and was trained by means of the Levenberg-Marquardt optimization algorithm (train$\mathrm{Im})$. The three main cut-off parameters (Vs, Vf, Ap) are the inputs, and the energy consumed (En) is the output. The sigmoid function is taken because of the behavior of the variables in relation to consumption, for the case studied was the one that obtained the best results.

\section{ANN training}

In order to establish a relationship between the input variables (cut-off parameters) with the output (energy consumption), a normalization function is used (Equation 1) that allows the database to be brought to values between 0.1 and 0.9 [10].

$x_{i}=0.8\left(\frac{x_{i}-x_{\min }}{x_{\text {max }}-x_{\min }}\right)+0.9$ 
Where $\mathrm{x}_{\min }$ and $\mathrm{x}_{\max }$ are the minimum and maximum value of each cutting parameter.

The number of neurons is determined by training the network, where the number of hidden layers is progressively increased until a coefficient of determination $\left(R^{2}\right)$ is achieved as close to 1 as possible, which would indicate that there is a linear correlation between predicted and simulated values, Equation 2 [11].

$R^{2}=1-\frac{\sum_{q=1}^{Q}\left(y_{q . \text { pred }}-y_{q . \exp }\right)^{2}}{\sum_{q=1}^{Q}\left(y_{q . \exp }-y_{m}\right)^{2}}$

Where $\mathrm{Q}$ is the number of data, $\mathrm{y}_{\mathrm{q}, \mathrm{pred}}$ represents the prediction of the network, $\mathrm{y}_{\mathrm{q}, \mathrm{exp}}$ the experimental response, $\mathrm{y}_{\mathrm{m}}$ the average of the current values and $\mathrm{q}$ the data that is being analyzed. At the end of the ANN training, the weights and the biases are obtained, the mentioned terms are necessary to create the equation that will allow the prediction of the energy consumption (Equation 3) which is constructed by the weighted sum of each neuron. The weights and adjustments are obtained through the trainlm algorithm which, by using a second-order approximation method, from a Hessian matrix, increasing the speed of the gradient and allows to find the coefficients of the function at low computational cost, being this an improved version of the Newton-Raphson method.

Out $t_{i}=\sum_{s}\left\{W_{o_{(1, s)}}\left[\frac{2}{1+e^{-2\left(b 1_{s}+\Sigma_{k} W_{i(s, k)} \mid n_{k}\right)}}-1\right]\right\}+b 2$

Where Out represents the predicted energy consumption, $W_{o}$ and $W_{i}$, the weights associated with each neuron and each variable, respectively, and b1 and b2 the biases, b1 being for each sigmoid transfer function and b2 being a single component that corresponds to the layer linear output.

\section{RESULTS AND DISCUSSION}

When the neural network was trained, a correlation of 0.9308 was found between the simulated data and the real data with a configuration that implements six neurons in the hidden layer, which relates 24 weights $\left(W_{i}=18\right.$; $\left.W_{o}=6\right)$ and seven biases $(b 1=6 ; b 2=1)$ to predict the energy consumption of the studied CNC machine.

Since the number of neurons is defined by a trial-and-er- ror system, it is necessary to establish a criterion to avoid overfitting [12], a very common error when training Feed-Forward type neural networks. This criterion is directly the value of R2 that, by allowing the software a maximum number of iterations in case of not finding the target, it is possible to validate which is the minimum number of neurons that are required to obtain a good correlation. In this case, the programmed algorithm showed that the optimal number of neurons is six and found a maximum correlation of 0.9308 in a total of 39190 iterations in a time of $11307 \mathrm{~s}$. Table 2 shows the parameters obtained that best suited the model for six neurons.

\section{Model validation}

The validation stage of the model is carried out after training. The value of $\mathrm{R}^{2}$ that was found by the algorithm was observed, and the regression is carried out between the simulated energy consumption data associated with equation 3 , with the energy consumption data from the experimental database. Figure 3 shows the regression graph of the Matlab® Toolbox ANN, where it is observed how far the experimental data are from the trained neural network model. Furthermore, the regression value found by the programmed algorithm coincides with the regression developed by the Toolbox, giving validity to the programmed convergence methods.

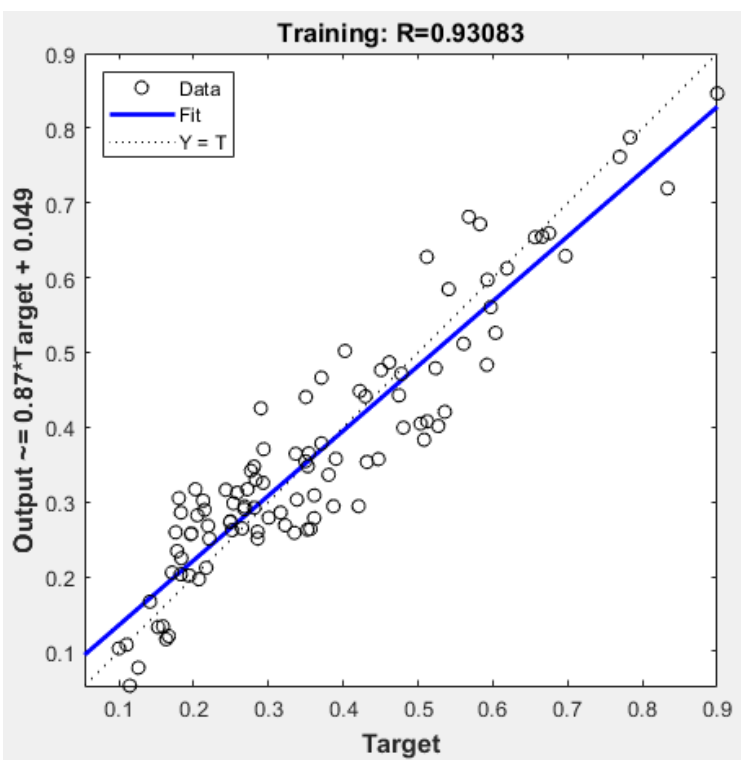

Figure 3: Regression graph of the Matlab®

Table 2: Weights and biases coefficients for the ANN analyzed

\begin{tabular}{|c|c|c|c|c|c|}
\hline & \multicolumn{3}{|c|}{$\mathrm{W}_{\mathrm{i}}$} & $\mathrm{W}_{\mathrm{o}}$ & $\mathrm{b} 1$ \\
\hline s1 & 0,02607 & 0,12670 & 4,09156 & $-1,54383$ & $-3,13000$ \\
\hline s2 & 30,67319 & 33,23736 & 32,95142 & 0,17930 & $-67,53917$ \\
\hline s3 & $-32,91900$ & $-33,08986$ & 1,50629 & $-0,22097$ & 18,75124 \\
\hline s4 & $-136,06209$ & 88,58675 & $-54,30884$ & 0,22780 & 20,86993 \\
\hline s5 & 20,20620 & $-14,22787$ & 0,60967 & 0,30072 & 2,39003 \\
\hline s6 & $-0,32266$ & $-0,00075$ & 3,30396 & 2,08438 & $-2,48333$ \\
\hline b2 & \multicolumn{5}{|c|}{0,66913} \\
\hline
\end{tabular}




\section{Sensitivity analysis}

According to Khataee and Mirzajani [13], it is possible to quantify the relative importance that each variable has on the studied model considering each input and using the weights obtained by the ANN, the relative importance is calculated using Equation 4.

$I_{j}=\frac{\sum_{\substack{m=N_{h} \\ m=1}}\left(\left(\left|W_{j_{m}}^{i_{n}}\right| / \sum_{\substack{k=N_{h} \\ k=1}}^{N_{h}} \mid \sum_{\substack{m=N_{h} \\ m=1}}^{m}\left(\left(\left|W_{j_{m}}^{i_{h}}\right| / \sum_{k=1}^{N_{h}}\left|W_{k_{m}}^{i_{h}}\right|\right) x\left|W_{m_{n}}^{h_{o}}\right|\right)\right.\right.}{\left.\left.W_{m_{n}}^{h_{o}} \mid\right)\right\}}$

Where $I_{j}$ is the relative importance of the $j$-th input variable, $N_{i}$ and $N_{h}$ are the number of inputs and hidden neurons, respectively. Finally, $\mathrm{W}$ represents the weights found. Figure 4 shows the relative influence of each variable. From it can be concluded that the depth per pass $A p$, is the variable that has the most influence on the energy consumption prediction model with $77 \%$ importance, this was expected because the deeper the greater forces the tool infringes on the part to be machined, on the other hand, the feed rate is the least relevant with a value of $9 \%$.

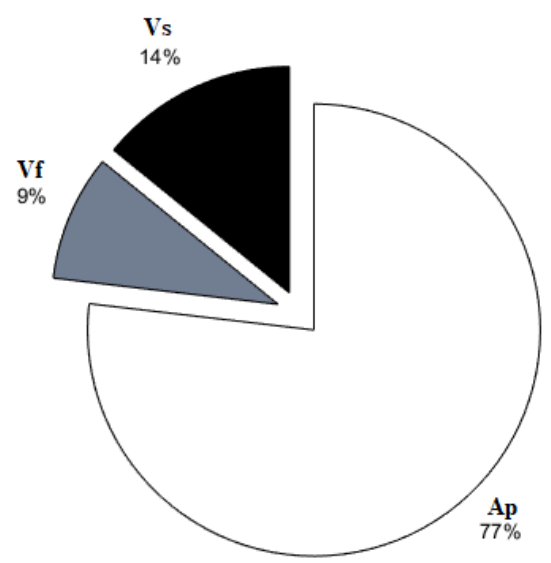

Figure 4: Relative importance of input variables in the energy consumption prediction. Being Vs the spindle speed, Vf the feed rate, and finally Ap the cutting depth

\section{CONCLUSIONS}

In this paper the prediction of the energy consumption media ANN was realized, using as input parameters the feed rate, depth of cut and spindle speed, finding that the depth of cut is the parameter which impact the most in the energy consumption with a $77 \%$ of relative importance. Results and validations show that the programed model was accurate for the problem abroad, obtaining an $\mathrm{R}^{2}$ of $93 \%$. Where errors in the prediction model could be related to the vibrations and wear of tool in the experiments.

The equation developed by the ANN allows to predict the energy consumption in the studied machining center departing from the input parameters, in this way it is possible to know the energy consumption related to a linear machining process with a $1 / 2$ " end mill, contributing to the study of the energy management in the manufacturing processes, allowing the optimization of cutting parameters, developing experimental mathematical models that helps to include the optimization techniques, depending from the different variables and tools purposed.

Artificial neural networks allow the development of mathematical models from experimental data, in which the physics problem can be described and therefore can be studied for parameter optimization processes, that could allow energy savings when finding the parameters that represent the lowest energy consumption for a given process. As has been shown in various works, to obtain an accurate prediction model requires a large amount of experimental data, so the coefficient of determination found could be higher if they were available, on the other hand, the human error at the time of data collection by experiments and wear on the tool can alter the associated consumption values, affecting the correlation of the data. Due to this last factor, is proposed future work, in addition to the study of the cutting parameters, also considering the wear on the tool to reduce the noise in the measurement of the energy consumption.

\section{ACKNOWLEDGEMENTS}

This work was supported by the Instituto Tecnológico Metropolitano de Medellín (Colombia), under the research line of Advanced Computing and Digital Design (CADD) and Mathematical Modelling, and Programming and Optimization Applied to Engineering, which belongs to the research group of Advanced Materials and Energy (MATyER). It was also supported by Universidad SurcoIombiana, and Institución Universitaria Pascual Bravo.

\section{CONFLICT OF INTEREST}

The authors declare that they have no conflict of interest.

\section{REFERENCES}

1. Hu, L., Peng, C., Evans, S., Peng, T. (2017) Minimising the machining energy consumption of a machine tool by sequencing the features of a part, Energy, vol. 121, pp. 292-305, doi: 10.1016/j.energy.2017.01.039.

2. Li, L., Li, C., Tang, Y., Li, L. (2018) Integration of process planning and cutting parameter optimization for energy-aware CNC machining, IEEE Int. Conf. Autom. Sci. Eng., vol. 2017-Augus, pp. 263-268, doi: 10.1109/COASE.2017.8256112.

3. Ma, F., Zhang, H., Cao, H., Hon, K. K. B. (2017) An energy consumption optimization strategy for CNC milling, Int. J. Adv. Manuf. Technol., vol. 90, no. 5-8, pp. 1715-1726, doi: 10.1007/s00170-016-9497-0.

4. Li, J., Salim, R. D., Aldlemy, M. S., Abdullah, J. M., Yaseen, Z. M. (2019) Fiberglass-Reinforced Polyester Composites Fatigue Prediction Using Novel Data-Intelligence Model, Arab. J. Sci. Eng., vol. 44, no. 4, pp. 3343-3356, Apr., doi: 10.1007/s13369-0183508-4.

Istraživanja i projektovanja za priverdu ISSN 1451-4117 Journal of Applied Engineering Science Vol. 20, No. 1,2022 
5. Xiangxue, W., Lunhui, X., Kaixun, C. (2019) Data-Driven Short-Term Forecasting for Urban Road Network Traffic Based on Data Processing and LSTM-RNN, Arab. J. Sci. Eng., vol. 44, no. 4, pp. 3043-3060, Apr., doi: 10.1007/s13369-018-3390-0.

6. Mori, M., Fujishima, M., Inamasu, Y., Oda, Y. (2011) A study on energy efficiency improvement for machine tools, CIRP Ann. - Manuf. Technol., vol. 60, no. 1, pp. 145-148, doi: 10.1016/j.cirp.2011.03.099.

7. Yan, J., Li, L. (2013) Multi-objective optimization of milling parameters-the trade-offs between energy, production rate and cutting quality, J. Clean. Prod., vol. 52, pp. 462-471, doi: 10.1016/j.jclepro.2013.02.030.

8. Jiang, Z., Zhou, F., Zhang, H., Wang, Y., Sutherland, J. W. (2015) Optimization of machining parameters considering minimum cutting fluid consumption," J. Clean. Prod., vol. 108, pp. 183-191, doi: 10.1016/j. jclepro.2015.06.007.

9. Calvanese, M. L., Albertelli, P., Matta, A., Taisch, M. (2013) Analysis of energy consumption in CNC machining centers and determination of optimal cutting conditions, Re-Engineering Manufacturing for Sustainability - Proceedings of the 20th CIRP International Conference on Life Cycle Engineering, pp. 227-232.

10. Hanafi, I., Cabrera, F. M., Dimane, F., Manzanares, J. T. (2015) Application of Particle Swarm Optimization for Optimizing the Process Parameters in Turning of PEEK CF30 Composites, Procedia Technol., vol. 22, no. October, pp. 195-202, 2016, doi: 10.1016/j.protcy.2016.01.044.
11. Harsha, N., Kumar, I. A., Raju, K. S. R., Rajesh, S. (2018) Prediction of Machinability characteristics of Ti6Al4V alloy using Neural Networks and Neuro-Fuzzy techniques, Mater. Today Proc., vol. 5, no. 2, pp. 8454-8463, doi: 10.1016/j.matpr.2017.11.541.

12. Karayel, D. (2009) Prediction and control of surface roughness in CNC lathe using artificial neural network, J. Mater. Process. Technol., vol. 209, no. 7, pp. 3125-3137, doi: 10.1016/j.jmatprotec.2008.07.023.

13. El-Mounayri, H., Kishawy, H., Briceno, J. (2005) Optimization of CNC ball end milling: A neural network-based model, J. Mater. Process. Technol., vol. 166, no. 1, pp. 50-62, doi: 10.1016/j.jmatprotec.2004.07.097.

14. Hernández, J. A., Rivera, W., Colorado, D., Moreno-Quintanar, G. (2012) Optimal COP prediction of a solar intermittent refrigeration system for ice production by means of direct and inverse artificial neural networks, Sol. Energy, vol. 86, no. 4, pp. 1108-1117, doi: 10.1016/j.solener.2011.12.021.

15. Khataee, A. R., Mirzajani (2010) O., UV/peroxydisulfate oxidation of C. I. Basic Blue 3: Modeling of key factors by artificial neural network, Desalination, vol. 251, no. 1-3, pp. 64-69, doi: 10.1016/j.desal.2009.09.142. 